\title{
THE LEVEL FOUR BRAID GROUP
}

\author{
TARA E. BRENDLE AND DAN MARGALIT
}

\begin{abstract}
By evaluating the Burau representation at $t=-1$, we obtain a symplectic representation of the braid group. We study the resulting congruence subgroups of the braid group, namely, the preimages of the principal congruence subgroups of the symplectic group. Our main result is that the level four congruence subgroup is equal to the group generated by squares of Dehn twists. We also show that the image of the Brunnian subgroup of the braid group under the symplectic representation is the level four congruence subgroup.
\end{abstract}

\section{INTRODUCTION}

The integral Burau representation of the braid group is the representation $\rho: \mathrm{B}_{n} \rightarrow \mathrm{GL}_{n}(\mathbb{Z})$ obtained by evaluating the (unreduced) Burau representation $\mathrm{B}_{n} \rightarrow \mathrm{GL}_{n}\left(\mathbb{Z}\left[t, t^{-1}\right]\right)$ at $t=-1$. The level $m$ congruence subgroup $\mathrm{B}_{n}[m]$ of $\mathbf{B}_{n}$ is the kernel of the mod $m$ reduction

$$
\mathrm{B}_{n} \stackrel{\rho}{\rightarrow} \mathrm{GL}_{n}(\mathbb{Z}) \rightarrow \mathrm{GL}_{n}(\mathbb{Z} / m) .
$$

As we explain in Section 2.1, $\rho$ can be regarded as a symplectic representation. Hence $\rho$ plays the same role for the braid group as the classical symplectic representation does for mapping class groups of closed surfaces. The representation $\rho$ has connections to many areas of mathematics, such as algebraic geometry, number theory, dynamics, and topology; see, e.g., the work A'Campo [1], Arnol'd [2], Smythe [29], Band-Boyland [5], Cohen-Wu [9], Funar-Kohno [14], Gambaudo-Ghys [15], Hain [16], Khovanov-Seidel [19], Magnus-Peluso [20], McMullen [21], Morifuji [24], Mumford [26], Venkataramana [31], Wajnryb [32], and $\mathrm{Yu}$ [34].

The mapping class group $\operatorname{Mod}(S)$ of a surface $S$ with marked points is the group of homotopy classes of homeomorphisms of $S$ fixing the set of marked points and fixing $\partial S$ pointwise. Let $D_{n}$ denote a closed disk with $n$ marked points in the interior. We have the following classical fact:

$$
\mathrm{B}_{n} \cong \operatorname{Mod}\left(D_{n}\right) \text {. }
$$

As such, it is natural to ask for descriptions of the $\mathrm{B}_{n}[\mathrm{~m}]$ that are intrinsic to either braid groups or mapping class groups. The first result in this direction is due to Arnol'd [2] who proved that $\mathrm{B}_{n}[2]$ is equal to the pure braid group $\mathrm{PB}_{n}$. Artin had previously proved that the latter is identified with the subgroup of $\operatorname{Mod}\left(D_{n}\right)$ generated by Dehn twists. Denote by $\mathcal{T}_{n}[m]$

The first author is supported in part by EPSRC grant EP/J019593/1. The second author supported by the National Science Foundation under Grant No. DMS - 1057874. 
the subgroup of $\operatorname{Mod}\left(D_{n}\right)$ generated by the $m$ th powers of all Dehn twists. Identifying $\mathrm{B}_{n}$ with $\operatorname{Mod}\left(D_{n}\right)$, we can summarize the theorems of Arnol'd and Artin as:

$$
\mathrm{B}_{n}[2]=\mathrm{PB}_{n}=\mathcal{T}_{n}[1] .
$$

Our main theorem gives an analogue for $\mathrm{B}_{n}[4]$. Let $\mathrm{PB}_{n}^{2}$ be the subgroup of $\mathrm{PB}_{n}$ generated by the squares of all elements; note that for any group $G$, the group $G^{2}$ equals the kernel of $G \rightarrow H_{1}(G ; \mathbb{Z} / 2)$.

Main Theorem. For $n \geq 1$, we have $\mathrm{B}_{n}[4]=\mathrm{PB}_{n}^{2}=\mathcal{T}_{n}[2]$.

The first equality of our Main Theorem, which is proven in Section 3, is well known in the case $n$ is odd; see, e.g., the unpublished paper of $\mathrm{Yu}$ [34]. This equality has a natural interpretation in terms of moduli spaces; see Section 2.

The second equality of our Main Theorem, proven in Section 4, has a precursor in the case of the mapping class group of a closed, orientable surface of genus $g$ : Humphries [17] proved that the level two mapping class group, that is, the kernel of the $\operatorname{map} \operatorname{Mod}\left(S_{g}\right) \rightarrow \mathrm{Sp}_{2 g}(\mathbb{Z} / 2)$ given by the action of $\operatorname{Mod}\left(S_{g}\right)$ on $H_{1}\left(S_{g} ; \mathbb{Z} / 2\right)$, is equal to the subgroup of $\operatorname{Mod}\left(S_{g}\right)$ generated by all squares of Dehn twists about nonseparating curves.

In Section 5 we determine the image under $\rho$ of the subgroup of $\mathrm{B}_{n}$ consisting of those braids that become trivial when any of the first $k$ strands are deleted; see Theorem 5.1 below. In the special case $k=n$ this is the Brunnian subgroup of $\mathrm{B}_{n}$ and in this case Theorem 5.1 says that the image under $\rho$ is precisely the level four congruence group $\operatorname{Sp}_{2 g}(\mathbb{Z})[4]$.

Related results. Besides the result of Humphries already mentioned, there are various other results about subgroups of $\mathrm{B}_{n}$ generated by powers of basic elements. Coxeter [10, Section 10] showed that the normal closure in $\mathrm{B}_{n}$ of the $m$ th power of any standard generator for $\mathrm{B}_{n}$ has finite index in $\mathrm{B}_{n}$ if and only if $1 / n+1 / m \leq 1 / 2$; see also [4]. Funar-Kohno [14, Theorem 1.1] proved that the intersection over $m$ of the groups $\mathcal{T}_{n}[m]$ is trivial. Humphries [18, Theorem 1] gave a complete description of when the group generated by (possibly differing) powers of Artin's (finitely many) generators for $\mathrm{PB}_{n}$ generates a subgroup of finite index; for instance, the group generated by the squares of Artin's generators has infinite index, in contrast to our Main Theorem.

Next, by evaluating the Burau representation at any $d$ th root of unity we obtain an analogue of the integral Burau representation. Building on work of McMullen [21], Venkataramana [31] showed that when $n / 2 \geq d \geq 3$ the image of $\mathrm{B}_{n}$ is arithmetic and is (up to finite index) as large as can be expected. Deligne-Mostow [11] previously gave analogues where the image is not arithmetic and Thurston [30] gave an interpretation of their work in terms of moduli spaces of convex polyhedra.

Finally, there is a more general notion of a congruence subgroup of the braid group. There is a natural action of $\mathrm{B}_{n}$ on the free group $F_{n}$, which 

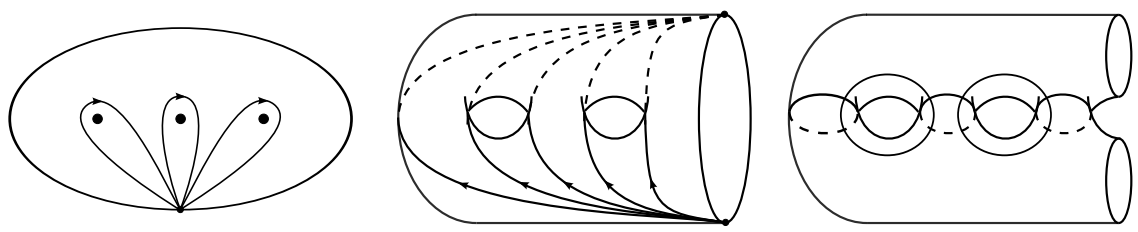

Figure 1. Left to right: the $\gamma_{i}$ in $D_{3}$, the lifts of the $\gamma_{i}$ to $X_{5}$, and the curves in $X_{6}$ whose Dehn twists lift the standard generators for $\mathrm{B}_{6}$

can be identified with the fundamental group of the disk with $n$ punctures. If $H$ is a characteristic subgroup of $F_{n}$, there is an induced homomorphism $\mathrm{B}_{n} \rightarrow \operatorname{Aut}\left(F_{n} / H\right)$ and the kernel is called a congruence subgroup of $\mathrm{B}_{n}$. It is a theorem of Asada [3] that every finite-index subgroup of $\mathrm{B}_{n}$ contains such a congruence subgroup; see also [12]. Thurston later gave a more elementary proof [22].

Acknowledgments. We would like to thank Joan Birman, Neil Fullarton, Louis Funar, Lalit Jain, Joseph Rabinoff, Douglas Ulmer, Kirsten Wickelgren, and Mante Zelvyte for helpful conversations. We would especially like to thank Andrew Putman, who pointed out a mistake in an earlier version and made several useful comments. We are also grateful to Jordan Ellenberg; a conversation with him on MathOverflow partly inspired the work in the last section.

\section{The Burau representation and a theorem of Arnol'D}

In this section we give a description of the integral Burau representation in terms of mapping class groups and also explain the classical result of Arnol'd that $\mathrm{B}_{n}[2]$ is equal to $\mathrm{PB}_{n}$. Then we give a reinterpretation of the first equality of our Main Theorem in terms of moduli spaces of points in $\mathbb{C}$.

2.1. The Burau representation. Let $\sigma_{1}, \ldots, \sigma_{n-1}$ denote the standard generators for $\mathrm{B}_{n}$. The (unreduced) Burau representation is the representation $\mathrm{B}_{n} \rightarrow \mathrm{GL}_{n}\left(\mathbb{Z}\left[t, t^{-1}\right]\right)$ defined by

$$
\sigma_{i} \mapsto I_{i-1} \oplus\left(\begin{array}{cc}
1-t & t \\
1 & 0
\end{array}\right) \oplus I_{n-i-1} .
$$

This representation obviously fixes the vector $(1,1, \ldots, 1)$ and this gives a 1-dimensional summand. The other summand is called the reduced Burau representation.

The Burau representation can also be described via topology. Let $D_{n}^{\circ}$ denote the punctured disk obtained from $D_{n}$ by removing the marked points and let $p \in \partial D_{n}^{\circ}$. Let $Y_{n}$ denote the universal abelian cover of $D_{n}^{\circ}$, let $t$ denote a generator for the deck transformation group, and let $\tilde{p}$ denote the full preimage of $p$. As a $\mathbb{Z}\left[t, t^{-1}\right]$-module, $H_{1}\left(Y_{n}, \tilde{p} ; \mathbb{Z}\right)$ has rank $n$; the generators are represented by path lifts to $Y_{n}$ of the loops $\gamma_{i}$ in $D_{n}^{\circ}$ shown in the left-hand side of Figure 1 (so the vector $(1,1, \ldots, 1)$ corresponds to 
a peripheral loop). Since the cover $Y_{n}$ is characteristic, each element of $\mathrm{B}_{n}$ induces a $t$-equivariant homeomorphism of $Y_{n}$ and the induced action on $H_{1}\left(Y_{n}, \tilde{p} ; \mathbb{Z}\right)$ is nothing other than the Burau representation.

The integral Burau representation. As mentioned, the integral Burau representation is the representation $\rho: \mathrm{B}_{n} \rightarrow \mathrm{GL}_{n}(\mathbb{Z})$ obtained by evaluating the Burau representation $\mathrm{B}_{n} \rightarrow \mathrm{GL}_{n}\left(\mathbb{Z}\left[t, t^{-1}\right]\right)$ at $t=-1$.

We can again describe this representation from the topological point of view. We consider the two-fold branched cover $X_{n} \rightarrow D_{n}$ with branch locus equal to the set of marked points. If $n=2 g+1$ then $X_{n}$ is a compact orientable surface $S_{g}^{1}$ of genus $g$ with one boundary component, and if $n=$ $2 g+2$ then $X_{n}$ is a compact orientable surface $S_{g}^{2}$ of genus $g$ with two boundary components.

Again because the (branched) cover $X_{n} \rightarrow D_{n}$ is characteristic, each element of $\operatorname{Mod}\left(D_{n}\right) \cong \mathrm{B}_{n}$ lifts to a (unique) element of $\operatorname{Mod}\left(X_{n}\right)$ and there is an induced homomorphism $\operatorname{Mod}\left(D_{n}\right) \rightarrow \operatorname{Mod}\left(X_{n}\right)$; denote the image by $\operatorname{SMod}\left(X_{n}\right)$. It is a special case of a theorem of Birman and Hilden [6] that this homomorphism is injective, but we will not use this fact.

Let $\tilde{p}=\left\{p_{1}, p_{2}\right\}$ be the preimage in $\partial X_{n}$ of $p$. Then $H_{1}\left(X_{n}, \tilde{p} ; \mathbb{Z}\right) \cong \mathbb{Z}^{n}$. Indeed, a basis for $H_{1}\left(X_{n}, \tilde{p} ; \mathbb{Z}\right)$ is given by the path lifts of the $\gamma_{i}$; see the middle of Figure 1. We claim that the composition

$$
\mathrm{B}_{n} \rightarrow \operatorname{Mod}\left(X_{n}\right) \rightarrow \operatorname{Aut}\left(H_{1}\left(X_{n}, \tilde{p} ; \mathbb{Z}\right)\right) \subseteq \mathrm{GL}_{n}(\mathbb{Z})
$$

is again the integral Burau representation. This can be easily checked by directly computing the action of each of the standard generators for $\mathrm{B}_{n}$. Alternatively, one can show that the kernel of the map $H_{1}\left(Y_{n}, \tilde{p} ; \mathbb{Z}\right) \rightarrow$ $H_{1}\left(X_{n}, \tilde{p} ; \mathbb{Z}\right)$ induced by the natural map $Y_{n} \rightarrow X_{n}$ is generated by elements of the form $t x+x$; this plus the fact that the lifts of an element of $\operatorname{Mod}\left(D_{n}\right)$ to $X_{n}$ and $Y_{n}$ are compatible gives the claim (the map $Y_{n} \rightarrow X_{n}$ is not surjective but is a covering map of $Y_{n}$ onto its image).

We can easily see from the latter description of the integral Burau representation that the reduced integral Burau representation of $\mathrm{B}_{2 g+1}$ is symplectic. Indeed, $H_{1}\left(S_{g}^{1}, \tilde{p} ; \mathbb{Z}\right)$ naturally splits as $H_{1}\left(S_{g}^{1} ; \mathbb{Z}\right) \oplus \mathbb{Z}$ and the first factor carries a symplectic form - the algebraic intersection number - which is preserved by $\mathrm{B}_{n}$. The algebraic intersection form on $H_{1}\left(S_{g}^{2}, \tilde{p} ; \mathbb{Z}\right)$ is already symplectic, and so the unreduced Burau representation of $\mathrm{B}_{2 g+2}$ is symplectic (and reducible).

Symplectic bases $\left(\vec{x}_{i}, \vec{y}_{i}\right)$ for both cases are shown in Figure 2 . The algebraic intersection number $\hat{\imath}\left(\vec{x}_{k}, \vec{y}_{k}\right)$ equals 1 for all $k$ and all other algebraic intersections between basis elements are zero. Throughout, we refer to these bases $\left\{\vec{x}_{i}, \vec{y}_{i}\right\}$ as the standard symplectic bases for $H_{1}\left(S_{g}^{1} ; \mathbb{Z}\right)$ and $H_{1}\left(S_{g}^{2}, \tilde{p} ; \mathbb{Z}\right)$. For the second case, notice that each boundary component represents the basis element $\vec{y}_{g+1}$ and so the integral Burau representation 


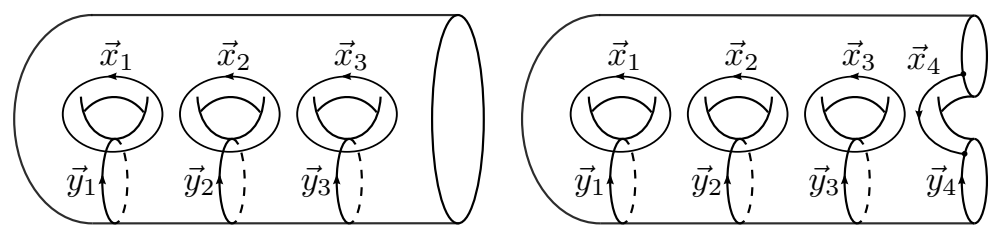

Figure 2. The standard symplectic bases for $H_{1}\left(S_{g}^{1} ; \mathbb{Z}\right)$ and $H_{1}\left(S_{g}^{2}, \tilde{p} ; \mathbb{Z}\right)$

can be regarded as a representation

$$
\rho: \mathrm{B}_{n} \rightarrow \begin{cases}\operatorname{Sp}_{2 g}(\mathbb{Z}) & n=2 g+1 \\ \left(\operatorname{Sp}_{2 g+2}(\mathbb{Z})\right)_{\vec{y}_{g+1}} & n=2 g+2\end{cases}
$$

(in the case $n=2 g+1$ we have dropped the trivial summand).

2.2. The pure braid group as a congruence subgroup. We now explain the theorem of Arnol'd that $\mathrm{PB}_{n}=\mathrm{B}_{n}[2]$. There is a canonical basis for $H_{1}\left(D_{n}^{\circ} ; \mathbb{Z} / 2\right)$ whose elements correspond to the $n$ punctures (these are represented by the $\gamma_{i}$ above). Let $H_{1}\left(D_{n}^{\circ} ; \mathbb{Z} / 2\right)^{\text {even }}$ denote the subspace consisting of elements with an even number of nonzero coordinates in the standard basis.

We would like to define a map $H_{1}\left(X_{n} ; \mathbb{Z} / 2\right) \rightarrow H_{1}\left(D_{n}^{\circ} ; \mathbb{Z} / 2\right)$ as follows: given a mod two cycle in $X_{n}$, we modify it by homotopy so that it avoids the fixed points of $\iota$ and then project to $D_{n}^{\circ}$. A priori this is not well defined, because homotopies in $X_{n}$ might push a cycle across a fixed point. Arnol'd proved that the map is indeed well defined [2, Lemma 1] and injective and that the image is $H_{1}\left(D_{n}^{\circ} ; \mathbb{Z} / 2\right)^{\text {even }}$ (see also [26, Lemma 8.12 and footnote on p. 145]). The key point is that a simple closed curve in $X_{n}$ surrounding a fixed point maps to zero in $H_{1}\left(D_{n}^{\circ} ; \mathbb{Z} / 2\right)$.

The isomorphism $H_{1}\left(D_{n}^{\circ} ; \mathbb{Z} / 2\right)^{\text {even }} \rightarrow H_{1}\left(X_{n} ; \mathbb{Z} / 2\right)$ is $\mathrm{B}_{n}$-equivariant, and so the elements of $\mathrm{B}_{n}$ that act trivially on $H_{1}\left(X_{n} ; \mathbb{Z} / 2\right)$ are exactly the ones that act trivially on $H_{1}\left(D_{n}^{\circ} ; \mathbb{Z} / 2\right)^{\text {even }}$. For $n \geq 3$ these are the braids that fix each marked point of $D_{n}$, namely, the pure braids. Thus $\mathrm{B}_{n}[2]=\mathrm{PB}_{n}$.

2.3. Moduli spaces. The first equality in our Main Theorem has an interpretation in terms of moduli spaces. Let $\mathcal{M}_{n}^{u}$ denote the moduli space of configurations of $n$ (unlabeled) points in $\mathbb{C}$. The double branched cover over such a configuration of points is an (open) hyperelliptic curve. Such a curve admits a unique hyperelliptic involution, and so we can regard $\mathcal{M}_{n}^{u}$ as the moduli space of hyperelliptic curves. The fundamental group of this moduli space is $\mathrm{B}_{n}$.

Next, let $\mathcal{M}_{n}$ denote the configuration space of $n$ labeled points in $\mathbb{C}$. Because of the identification of $H_{1}\left(X_{n} ; \mathbb{Z} / 2\right)$ with $H_{1}\left(D_{n}^{\circ} ; \mathbb{Z} / 2\right)^{\text {even }}$, the ordering of the points in some configuration of points gives rise to a basis for the mod two homology of the associated hyperelliptic curve, namely, the differences of consecutive points in the configuration. The fundamental 
group of $\mathcal{M}_{n}$ is $\mathrm{PB}_{n}$ and the forgetful map $\mathcal{M}_{n} \rightarrow \mathcal{M}_{n}^{u}$ is the covering map associated to the inclusion $\mathrm{PB}_{n} \rightarrow \mathrm{B}_{n}$.

Now let $n \geq 3$ and let $m$ be any positive even integer. Given a point in $\mathcal{M}_{n}$, we may consider the associated open hyperelliptic curve $X$. A hyperelliptic level $m$ marking of $X$ is a basis for $H_{1}(X ; \mathbb{Z} / m)$ whose $\bmod$ two reduction is the canonical one given in the previous paragraph. Let $\mathcal{M}_{n}[m]$ denote the moduli space of open hyperelliptic curves as above with hyperelliptic level $m$ markings (so $\mathcal{M}_{n}[2]=\mathcal{M}_{n}$ ). The space $\mathcal{M}_{n}[m]$ is connected and has fundamental group $\mathrm{B}_{n}[m]$. The forgetful map $\mathcal{M}_{n}[m] \rightarrow$ $\mathcal{M}_{n}$ is the covering map corresponding to the inclusion $\mathrm{B}_{n}[m] \rightarrow \mathrm{PB}_{n}$.

Since $\mathrm{PB}_{n} / \mathrm{PB}_{n}^{2}=\mathrm{PB}_{n} / \mathrm{B}_{n}[4]$ is the universal 2-primary abelian quotient of $\mathrm{PB}_{n}$ we obtain the following corollary, also observed by $\mathrm{Yu}[34$, Corollary 7.4] in the case $n$ odd.

Corollary 2.1. For $n \geq 3$, the covering space $\mathcal{M}_{n}[4] \rightarrow \mathcal{M}_{n}$ is universal among 2-primary covering spaces of $\mathcal{M}_{n}$.

The space $\mathcal{M}_{n}[4]$ has an algebro-geometric description as follows:

$$
\text { Spec } \mathbb{C}\left[t_{i}: 1 \leq i \leq n\right]\left[\left(t_{i}-t_{j}\right)^{-1}, \sqrt{t_{i}-t_{j}}: 1 \leq i<j \leq n\right] ;
$$

this is the so-called Kümmer cover of $\mathcal{M}_{n}$. That these two covering spaces are isomorphic follows, for instance, from Corollary 2.1 and the fact that the deck groups are the same.

\section{LEVEL FOUR VERSUS THE MOD TWO KERNEL}

The goal of this section is to prove the following proposition, which is one half of our Main Theorem.

Proposition 3.1. For any $n$, we have $\mathrm{B}_{n}[4]=\mathrm{PB}_{n}^{2}$.

For the case of $n=2 g+1$, Proposition 3.1 follows easily from the wellknown facts Theorem 3.3(1) and Lemma 3.4(1) below. As mentioned, in this case the observation was already made by $\mathrm{Yu}$ [34, Proof of Corollary 7.4]. Most of the work in this section is devoted to proving the analogs of these results for the case of $n=2 g+2$, namely Theorem 3.3(2) and Lemma 3.4(2).

Throughout this section, denote the symplectic form on $\mathbb{Z}^{2 g}$ by $\hat{\imath}$ and fix a symplectic basis $\mathcal{B}_{g}=\left\{\vec{x}_{1}, \vec{y}_{1}, \ldots, \vec{x}_{g}, \vec{y}_{g}\right\}$ with $\hat{\imath}\left(\vec{x}_{k}, \vec{y}_{k}\right)=1$ for all $k$.

Symplectic transvections. The symplectic transvection associated to $\vec{v} \in$ $\mathbb{Z}^{2 g}$ is the linear transformation $\tau_{\vec{v}}: \mathbb{Z}^{2 g} \rightarrow \mathbb{Z}^{2 g}$ given by

$$
\tau_{\vec{v}}(\vec{w})=\vec{w}+\hat{\imath}(\vec{w}, \vec{v}) \vec{v} .
$$

If $c$ is a simple closed curve in $S_{g}^{1}$ that with some choice of orientation represents $\vec{v} \in H_{1}\left(S_{g}^{1} ; \mathbb{Z}\right)$, then the image of the Dehn twist $T_{c}$ in $\operatorname{Sp}_{2 g}(\mathbb{Z})$ is the transvection $\tau_{\vec{v}}$ (this makes sense because $\tau_{\vec{v}}=\tau_{-\vec{v}}$ ).

The first statement of the next proposition is a slight variation of a classical theorem found, for instance, in the book by Mumford [25, Proposition 
A3]. (The modified generating set will make our pictures simpler in the proof of Theorem 3.3.)

Proposition 3.2. Let $g \geq 2$.

(1) The group $\mathrm{Sp}_{2 g}(\mathbb{Z})[2]$ is generated by the $\tau_{\vec{v}}^{2}$ with $\vec{v}$ in

$$
\left\{\vec{x}_{i}\right\} \cup\left\{\vec{y}_{j}\right\} \cup\left\{\vec{x}_{i}+\vec{x}_{j}\right\} \cup\left\{\vec{y}_{i}-\vec{y}_{j}\right\} \cup\left\{\vec{x}_{i}-\vec{y}_{j}\right\} .
$$

(2) The group $\left(\operatorname{Sp}_{2 g+2}(\mathbb{Z})[2]\right)_{\vec{y}_{g+1}}$ is generated by the $\tau_{\vec{v}}^{2}$ with $\vec{v}$ in $\left\{\vec{x}_{i} \mid i \neq g+1\right\} \cup\left\{\vec{y}_{j}\right\} \cup\left\{\vec{x}_{i}+\vec{x}_{j} \mid i, j \neq g+1\right\} \cup\left\{\vec{y}_{i}-\vec{y}_{j}\right\} \cup\left\{\vec{x}_{i}-\vec{y}_{j} \mid i \neq g+1\right\}$.

Proof. According to Mumford, $\operatorname{Sp}_{2 g}(\mathbb{Z})[2]$ is generated by the $\tau_{\vec{v}}^{2}$ with $\vec{v}$ in

$$
\mathcal{B}_{g} \cup\left\{\vec{u}+\vec{w} \mid \vec{u}, \vec{w} \in \mathcal{B}_{g}, \vec{u} \neq \vec{w}\right\} .
$$

For the first statement it suffices to show that we can replace the $\vec{y}_{i}+\vec{y}_{j}$ and $\vec{x}_{i}+\vec{y}_{j}$ in this generating set with the corresponding $\vec{y}_{i}-\vec{y}_{j}$ and $\vec{x}_{i}-\vec{y}_{j}$.

The first observation is that

$$
\tau_{\vec{x}_{i}}^{2} \tau_{\vec{x}_{i}+\vec{y}_{i}}^{2} \tau_{\vec{x}_{i}}^{-2}=\tau_{\vec{x}_{i}-\vec{y}_{i}}^{2}
$$

and so we may replace the $\vec{x}_{i}+\vec{y}_{i}$ with the corresponding $\vec{x}_{i}-\vec{y}_{i}$.

Next, we note that $\omega_{i}=\tau_{\vec{x}_{i}}^{2} \tau_{\vec{y}_{i}}^{2} \tau_{\vec{x}_{i}-\vec{y}_{i}}^{2}$ negates $\vec{x}_{i}$ and $\vec{y}_{i}$ while fixing all other elements of $\mathcal{B}_{g}$. It follows that for $i \neq j$ we have

$$
\begin{aligned}
& \omega_{j} \tau_{\vec{y}_{i}+\vec{y}_{j}}^{2} \omega_{j}^{-1}=\tau_{\vec{y}_{i}-\vec{y}_{j}}^{2} \text { and } \\
& \omega_{j} \tau_{\vec{x}_{i}+\vec{y}_{j}}^{2} \omega_{j}^{-1}=\tau_{\vec{x}_{i}-\vec{y}_{j}}^{2},
\end{aligned}
$$

and so we can replace the $\vec{y}_{i}+\vec{y}_{j}$ and $\vec{x}_{i}+\vec{y}_{j}$ with $\vec{y}_{i}-\vec{y}_{j}$ and $\vec{x}_{i}-\vec{y}_{j}$, as desired.

We proceed to the second statement. Let $M \in\left(\operatorname{Sp}_{2 g+2}(\mathbb{Z})[2]\right)_{\vec{y}_{g+1}}$. Since $M$ fixes $\vec{y}_{g+1}$, is the identity modulo two, and preserves $\hat{\imath}\left(\vec{x}_{g+1}, \vec{y}_{g+1}\right)$, we have

$$
M\left(\vec{x}_{g+1}\right)=\left(2 c_{1} \vec{x}_{1}+2 d_{1} \vec{y}_{1}+\cdots+2 c_{g} \vec{x}_{g}+2 d_{g} \vec{y}_{g}\right)+\vec{x}_{g+1}+2 d_{g+1} \vec{y}_{g+1}
$$

for some $c_{1}, d_{1}, \ldots, c_{g}, d_{g}, d_{g+1} \in \mathbb{Z}$. If for $i<g+1$ we apply the product $\tau_{\vec{y}_{g+1}-\vec{x}_{i}}^{-2 c_{i}} \tau_{\vec{x}_{i}}^{2 c_{i}}$ or $\tau_{\vec{y}_{g+1}-\vec{y}_{i}}^{-2 d_{i}} \tau_{\vec{y}_{i}}^{2 d_{i}}$, the effect is to eliminate the corresponding term $2 c_{i} \vec{x}_{i}$ or $2 d_{i} \vec{y}_{i}$ at the expense of changing the coeffidcient $d_{g+1}$. We thus reduce to the case where $M\left(\vec{x}_{g+1}\right)$ lies in $\left\langle\vec{x}_{g+1}, \vec{y}_{g+1}\right\rangle$ and where $M$ fixes $\vec{y}_{g+1}$. By then applying a power of $\tau_{\vec{y}_{g+1}}^{2}$ we reduce to the case that $M$ fixes both $\vec{x}_{g+1}$ and $\vec{y}_{g+1}$.

Since $M$ preserves the symplectic form and fixes $\left\langle\vec{x}_{g+1}, \vec{y}_{g+1}\right\rangle$ it follows that $M$ preserves $\left\langle\vec{x}_{1}, \vec{y}_{1}, \ldots, \vec{x}_{g}, \vec{y}_{g}\right\rangle$, that is, $M$ decomposes as a direct sum $M_{g} \oplus I_{2}$, where $M_{g}$ is the action on $\left\langle\vec{x}_{1}, \vec{y}_{1}, \ldots, \vec{x}_{g}, \vec{y}_{g}\right\rangle$. Since the image of the generating set from the first statement under the inclusion $\mathrm{Sp}_{2 g}(\mathbb{Z}) \rightarrow\left(\operatorname{Sp}_{2 g+2}(\mathbb{Z})[2]\right)_{\vec{y}_{g+1}}$ is contained in the generating set from the second statement, the proposition follows. 
It follows from the computation of the abelianization of $\operatorname{Sp}_{2 g}(\mathbb{Z})[2]$ below that the generating set for $\mathrm{Sp}_{2 g}(\mathbb{Z})$ in Proposition 3.2 is minimal. See the paper of Church and Putman [8] for minimal generating sets for the other congruence subgroups of $\mathrm{Sp}_{2 g}(\mathbb{Z})$.

The first statement of the following theorem is due to A'Campo [1, Théorème 1] (see also Mumford [26, Lemma 8.12] and Wajnryb [32, Theorem 1]).

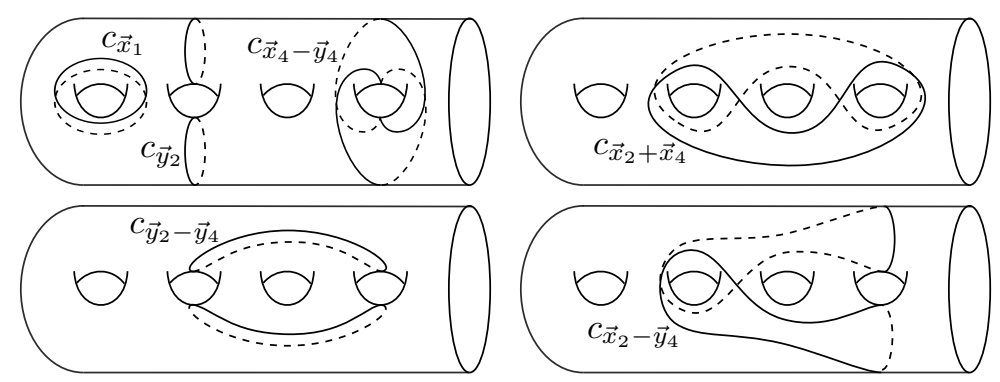

Figure 3. The curves used in the proof of Theorem 3.3

Theorem 3.3. Let $g \geq 2$.

(1) The restriction $\rho: \mathrm{PB}_{2 g+1} \rightarrow \mathrm{Sp}_{2 g}(\mathbb{Z})[2]$ is surjective.

(2) The restriction $\rho: \mathrm{PB}_{2 g+2} \rightarrow\left(\mathrm{Sp}_{2 g+2}(\mathbb{Z})[2]\right)_{\vec{y}_{g+1}}$ is surjective.

Proof of Theorem 3.3. It suffices to realize each generator from parts (1) and (2) of Proposition 3.2. For each transvection $\tau_{\vec{v}}$ as in the proposition we can find a simple closed curve $c_{\vec{v}}$ lying in $S_{g}^{1}$ or $S_{g}^{2}$ accordingly and with the following properties:

(1) for some choice of orientation of $c_{\vec{v}}$, we have $\left[\vec{c}_{\vec{v}}\right]=\vec{v}$ and

(2) $\iota\left(c_{\vec{v}}\right) \cap c_{\vec{v}}=\emptyset$.

The required curves are shown in Figure 3 (for the second statement of the theorem we should imagine $S_{g}^{2}$ as lying inside $S_{g+1}^{1}$ and check that the required curves avoid $S_{g+1} \backslash S_{g}^{2}$ ). It follows from the second condition that $T_{c_{\vec{v}}} T_{\iota\left(c_{\vec{v}}\right)}$ lies in $\operatorname{SMod}\left(S_{g}^{1}\right)$ or $\operatorname{SMod}\left(S_{g}^{2}\right)$, and hence corresponds an element of the appropriate pure braid group $\left(\mathrm{PB}_{2 g+1}\right.$ or $\left.\mathrm{PB}_{2 g+2}\right)$. By the first condition, the image of this product in the appropriate symplectic group is $\tau_{\vec{v}}^{2}$, as desired.

The image of the full braid group. We pause to record a (well-known) consequence of Theorem 3.3. Fix $g \geq 1$ and let $n=2 g+1$. Let $\Sigma_{n}$ denote the symmetric group on $n$ letters. The action of $\Sigma_{n}$ on the marked points of $D_{n}$ induces an action of $\Sigma_{n}$ on $H_{1}\left(D_{n}^{\circ} ; \mathbb{Z} / 2\right) \cong H_{1}\left(X_{n} ; \mathbb{Z} / 2\right)$ and hence an embedding $\Sigma_{n} \rightarrow \operatorname{Sp}_{2 g}(\mathbb{Z} / 2)$ with image a group of permutation matrices. Each such matrix has a natural lift to $\operatorname{Sp}_{2 g}(\mathbb{Z})$ and this gives rise to an 
action of $\Sigma_{n}$ on $\operatorname{Sp}_{2 g}(\mathbb{Z})[2]$ by conjugation. There are analogous statements for $\operatorname{Sp}_{2 g+2}(\mathbb{Z})$ when $n=2 g+2$. We thus have:

$$
\begin{aligned}
& \rho\left(\mathrm{B}_{2 g+1}\right) \cong \mathrm{Sp}_{2 g}(\mathbb{Z})[2] \rtimes \Sigma_{2 g+1} \\
& \rho\left(\mathrm{B}_{2 g+2}\right) \cong\left(\mathrm{Sp}_{2 g+2}(\mathbb{Z})[2]\right)_{\vec{y}_{g+1}} \rtimes \Sigma_{2 g+2} .
\end{aligned}
$$

See Wajnryb [32, page 147] for an interpretation of $\rho\left(\mathrm{B}_{n}\right)$ in terms of quadratic forms on $\mathbb{Z}^{2 g}$.

In particular, the group $\rho\left(\mathrm{B}_{2 g+1}\right)$ contains the square of each transvection in $\operatorname{Sp}_{2 g}(\mathbb{Z})$ and so the mod $p$ reduction contains every transvection if $p$ is odd. Wajnryb [33] used this plus the fact that $\operatorname{Sp}_{2 g}(\mathbb{Z} / p)$ is generated by transvections in order to give a simple presentation for the latter (and similarly for $\mathrm{B}_{2 g+2}$ ).

The symplectic Lie algebra. Let $J$ denote the $2 g \times 2 g$ matrix associated to the symplectic form on $\mathbb{Z}^{2 g}$ and let $j$ denote the mod 2 reduction. Just as $\operatorname{Sp}_{2 g}(\mathbb{Z})$ is the group of integral matrices that satisfy $M J=J M^{T}$, the group $\mathfrak{s p}_{2 g}(\mathbb{Z} / 2)$ is the additive group of $2 g \times 2 g$ matrices $m$ with entries in $\mathbb{Z} / 2$ and with $m j=j m^{T}$. If we reorder the symplectic basis for $\mathbb{Z}^{2 g}$ as $\left(\vec{x}_{1}, \ldots, \vec{x}_{g}, \vec{y}_{g}, \ldots, \vec{y}_{1}\right)$, then $\mathfrak{s p}_{2 g}(\mathbb{Z} / 2)$ is the set of matrices over $\mathbb{Z} / 2$ that are persymmetric (symmetric along the anti-diagonal). To put it yet another way, these are the matrices where, for each $\vec{v}$ and $\vec{w}$ in the standard basis, the $\vec{v} \vec{w}$-entry is equal to the $\vec{w}^{\star} \vec{v}^{\star}$-entry (really these are the entries corresponding to the mod two reductions of those vectors).

For $\vec{v}, \vec{w} \in \mathcal{B}_{g}$, let $m_{\vec{v} \vec{w}}$ be the element of $\mathfrak{s p}_{2 g}(\mathbb{Z} / 2)$ obtained from the zero matrix by replacing the $\vec{v} \vec{w}$ - and $\vec{w}^{\star} \vec{v}^{\star}$-entries with 1 (if $\vec{v}=\vec{w}^{\star}$ this matrix has a single nonzero entry). From the definition, we see that $m_{\vec{v} \vec{w}}=m_{\vec{w}^{\star} \vec{v}^{\star}}$. Clearly the $m_{\vec{v} \vec{w}}$ generate the abelian group $\mathfrak{s p}_{2 g}(\mathbb{Z} / 2)$.

We remark that there is an isomorphism $\mathfrak{s p}_{2 g}(\mathbb{Z} / 2) \rightarrow S^{2}\left((\mathbb{Z} / 2)^{2 g}\right)$ given by $m_{\vec{v} \vec{w}} \mapsto \vec{v} \vec{w}^{\star}$. From this or any of the other descriptions of $\mathfrak{s p}_{2 g}(\mathbb{Z} / 2)$, we can easily check that $\mathfrak{s p}_{2 g}(\mathbb{Z} / 2)$ is isomorphic to $(\mathbb{Z} / 2)\left(\begin{array}{c}2 g+1 \\ 2\end{array}\right)$.

The abelianization of the symplectic group. There is a homomorphism

$$
\begin{aligned}
\psi: \operatorname{Sp}_{2 g}(\mathbb{Z})[2] & \rightarrow \mathfrak{s p}_{2 g}(\mathbb{Z} / 2) \\
I_{2 g}+2 A & \mapsto A \bmod 2 .
\end{aligned}
$$

Evidently, the kernel of $\psi$ is $\operatorname{Sp}_{2 g}(\mathbb{Z})[4]$. It is well known that $\psi$ is surjective and it is a theorem of Newman-Smart that $\psi$ is in fact the abelianization of $\mathrm{Sp}_{2 g}(\mathbb{Z})[2]$, that is, $\mathrm{Sp}_{2 g}(\mathbb{Z})[4]$ is the commutator subgroup [27, Theorem 7] (this generalizes to higher levels; see [28, Section 3.1] for a survey). In particular, there is a short exact sequence

$$
1 \rightarrow \operatorname{Sp}_{2 g}(\mathbb{Z})[4] \rightarrow \operatorname{Sp}_{2 g}(\mathbb{Z})[2] \stackrel{\psi}{\rightarrow} \mathfrak{s p}_{2 g}(\mathbb{Z} / 2) \rightarrow 1
$$


We will need to describe the image of $\left(\operatorname{Sp}_{2 g+2}(\mathbb{Z})[2]\right)_{\vec{y}_{g+1}}$ under $\psi$. For any $\vec{v} \in(\mathbb{Z} / 2)^{2 g}$, set

$$
\operatorname{Ann}(\vec{v})=\left\{m \in \mathfrak{s p}_{2 g+2}(\mathbb{Z} / 2) \mid m(\vec{v})=0\right\} .
$$

It is straightforward to check that the image of $\left(\operatorname{Sp}_{2 g+2}(\mathbb{Z})[2]\right)_{\vec{y}_{g+1}}$ under $\psi$ lies in $\operatorname{Ann}\left(\vec{y}_{g+1}\right)$ and that $\operatorname{Ann}\left(\vec{y}_{g+1}\right)$ is generated by

$$
\left\{m_{\vec{v} \vec{w}} \mid \vec{v}, \vec{w} \in\left\{\vec{x}_{1}, \vec{y}_{1}, \ldots, \vec{x}_{g+1}, \vec{y}_{g+1}\right\}, \vec{v} \neq \vec{x}_{g+1}, \vec{w} \neq \vec{y}_{g+1}\right\} .
$$

In particular, $\operatorname{Ann}\left(\vec{y}_{g+1}\right)$ is isomorphic to $(\mathbb{Z} / 2)\left(\begin{array}{c}2 g+2 \\ 2\end{array}\right)$.

Lemma 3.4. Let $g \geq 0$. We have:

(1) The map $\psi: \operatorname{Sp}_{2 g}(\mathbb{Z})[2] \rightarrow \mathfrak{s p}_{2 g}(\mathbb{Z} / 2)$ is surjective, and

(2) The map $\psi:\left(\operatorname{Sp}_{2 g+2}(\mathbb{Z})[2]\right)_{\vec{y}_{g+1}} \rightarrow \operatorname{Ann}\left(\vec{y}_{g+1}\right)$ is surjective.

Proof. As we already said, the first statement is well known. However, we give a proof for completeness and to establish some notation needed for the second case. Specifically, for any choice of $\vec{v}$ and $\vec{w}$ in the standard basis $\left\{\vec{x}_{1}, \vec{y}_{1}, \ldots, \vec{x}_{g}, \vec{y}_{g}\right\}$, we would like to define an element $M_{\vec{v} \vec{w}}$ of $\operatorname{Sp}_{2 g}(\mathbb{Z})[2]$ whose image is the matrix $m_{\vec{v} \vec{w}}$ given above. Let $N_{\vec{v}}$ denote the matrix obtained from the identity by negating the $\vec{v} \vec{v}$ - and $\vec{v}^{\star} \vec{v}^{\star}$-entries. We set

$$
M_{\vec{v} \vec{w}}= \begin{cases}N_{\vec{v}} & \vec{v}=\vec{w} \\ \tau_{\vec{v}}^{2} & \vec{v}=\vec{w}^{\star} \\ \tau_{\vec{w}^{\star}+\vec{v}^{2}}^{-2} \tau_{\vec{w}^{\star}}^{2} \tau_{\vec{v}}^{2} & \text { otherwise. }\end{cases}
$$

It is straightforward to check that the image of each $N_{\vec{v} \vec{w}}$ is the desired matrix $m_{\vec{v} \vec{w}}$. Since the $m_{\vec{v} \vec{w}}$ generate $\mathfrak{s p}_{2 g}(\mathbb{Z} / 2)$, the first statement follows.

We already said that the $m_{\vec{v} \vec{w}}$ with $\vec{v} \neq \vec{x}_{g+1}$ and $\vec{w} \neq \vec{y}_{g+1}$ generate Ann $\left(\vec{y}_{g+1}\right)$ and so for the second statement it suffices to note that the corresponding matrices $M_{\vec{v} \vec{w}}$ lie in $\left(\mathrm{Sp}_{2 g+2}(\mathbb{Z})[2]\right)_{\vec{y}_{g+1}}$, which follows immediately from the definitions.

Proof of Proposition 3.1. First we treat the case of $n=2 g+1$. Any map from a group to a 2-primary abelian group factors through its universal $\mathbb{Z} / 2$-vector space quotient, and so there is a commutative diagram

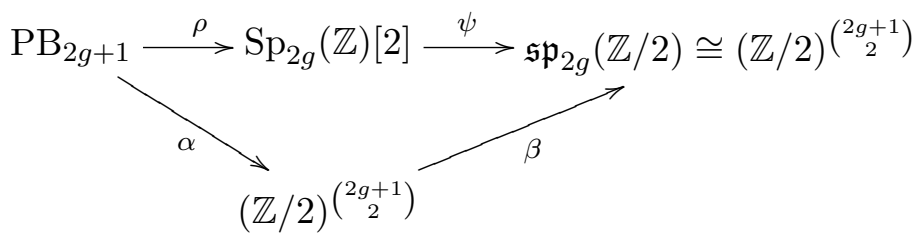

Since $\psi \circ \rho$ is surjective (Theorem 3.3(1) and Lemma 3.4(1)), it follows that $\beta$ is an isomorphism. Thus $\operatorname{ker}(\psi \circ \rho)=\operatorname{ker} \alpha$. We already said that $\operatorname{ker} \alpha=$ $\mathrm{PB}_{2 g+1}^{2}$. Since ker $\psi=\mathrm{Sp}_{2 g}(\mathbb{Z})[4]$ and $\mathrm{B}_{2 g+1}[4] \subseteq \mathrm{B}_{2 g+1}[2]=\mathrm{PB}_{2 g+1}$ we have $\operatorname{ker}(\psi \circ \rho)=\mathrm{B}_{2 g+1}[4]$. This completes the proof in the case $n$ odd. 
For $n=2 g+2$ even the proof is the same except that we use Theorem 3.3(2), Lemma 3.4(2), and the fact that $\operatorname{Ann}\left(\vec{y}_{g+1}\right) \cong(\mathbb{Z} / 2)\left(\begin{array}{c}(2 g+2 \\ 2\end{array}\right)$.

\section{Squares of twists Versus the mod tWo Kernel}

Combined with Proposition 3.1 the following proposition gives the Main Theorem.

Proposition 4.1. For any $n$ we have $\mathcal{T}_{n}[2]=\mathrm{PB}_{n}^{2}$.

In order to prove Proposition 4.1, we need a certain relation amongst Dehn twists in $D_{3}$. The configuration of curves involved in this relation is reminiscent of the lantern relation and most of the twists involved are squared; hence we refer to it as the squared lantern relation.

Proposition 4.2 (The squared lantern relation). Let $a, b, c, d$, and $e$ be the curves in $D_{3}$ shown in Figure 4. The following relation holds in $\operatorname{Mod}\left(D_{3}\right)$ :

$$
\left[T_{a}, T_{b}\right]=T_{a}^{2} T_{d}^{2} T_{c}^{2} T_{e}^{-2} .
$$
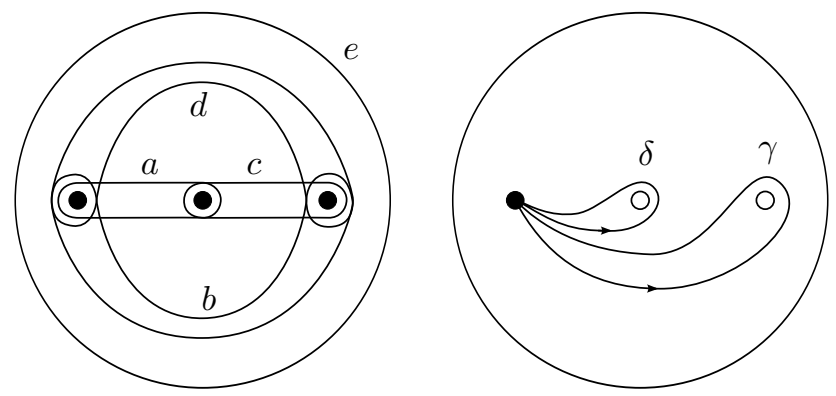

FiguRE 4. The curves in the squared lantern relation and the loops used in the proof

Let $\sigma_{1}, \ldots, \sigma_{n-1}$ denote the standard generators for the braid group $\mathrm{B}_{n}$. For $1 \leq i<j \leq n$ let $a_{i j}=\omega^{-1} \sigma_{i}^{2} \omega$, where $\omega=\sigma_{i+1} \cdots \sigma_{j-1}$ (elements of $\mathrm{B}_{n}$ are composed left to right). Artin proved that the $a_{i j}$ generate $\mathrm{PB}_{n}$.

Each $a_{i j}$ is equal to a Dehn twist about a curve $c_{i j}$ in $D_{n}$ surrounding two marked points. If we place the marked points of $D_{n}$ in a horizontal line, and if we choose the $\sigma_{i}$ to be right-handed half-twists, then $c_{i j}$ is the boundary of a regular neighborhood of an arc whose interior lies below the line and connects the $i$ th marked point to the $j$ th.

We can reinterpret the squared lantern relation in terms of Artin's generators as follows:

$$
\left[a_{12}, a_{13}\right]=a_{12}^{2}\left(a_{12}^{-1} a_{13}^{2} a_{12}\right) a_{23}^{2}\left(a_{13} a_{12} a_{23}\right)^{-2}
$$

(in the braid group elements are multiplied left to right). 
Push maps. While Proposition 4.2 can be verified using any of the standard solutions to the word problem for either the mapping class group, the braid group, or the pure braid group, we will give here a conceptual proof. The ideas we develop here will also be used in the next section.

Choose one marked point of $D_{n}$, call it $p$, and delete the other $n-1$ marked points from $D_{n}$. Denote the resulting disk with $n-1$ punctures and one marked point by $D_{n}^{\prime}$. There is a push map:

$$
\Psi: \pi_{1}\left(D_{n}^{\prime}, p\right) \rightarrow \operatorname{PMod}\left(D_{n}\right) \cong \mathrm{PB}_{n}
$$

defined as follows. Given $\gamma \in \pi_{1}\left(D_{n}^{\prime}, p\right)$, we choose an isotopy of $p$ that pushes $p$ along $\gamma$ and we extend this to an isotopy of $D_{n}^{\prime}$. At the end of the isotopy there is an induced homeomorphism of $D_{n}^{\prime}$, hence $D_{n}$, whose homotopy class is $\Psi(\gamma)$ (see [13, Section 4.2] for details).

If $\gamma$ has a simple representative $w$ with regular neighborhood $A$ in $D_{n}^{\prime}$, then $\Psi(\gamma)$ is equal to the product $T_{\ell} T_{r}^{-1}$, where $\ell$ and $r$ denote the components of $\partial A$ lying to the left and right of $\gamma$, respectively (see [13, Fact 4.7]). It is sometimes the case that one of $\ell$ or $r$ is inessential, in which case we can omit the corresponding trivial Dehn twist. Since products in $\pi_{1}\left(D_{n}^{\prime}, p\right)$ are usually written left to right, the map $\Psi$ is an antihomomorphism.

Proof of Proposition 4.2. Choose the marked point $p$ as in the right-hand side of Figure 4. As above there is a map $\Psi: \pi_{1}\left(D_{3}^{\prime}, p\right) \rightarrow \operatorname{PMod}\left(D_{3}\right)$. Let $\gamma$ and $\delta$ be the two elements of $\pi_{1}\left(D_{3}^{\prime}, p\right)$ indicated in the same figure; these generate the free group $\pi_{1}\left(D_{3}^{\prime}, p\right) \cong F_{2}$. As above we have

$$
\Psi(\gamma)=T_{b}^{-1}, \quad \Psi(\delta)=T_{a}^{-1}, \text { and } \Psi(\gamma \delta)=T_{c} T_{e}^{-1} .
$$

In the free group $\pi_{1}\left(D_{3}^{\prime}, p\right)$, we can write

$$
[\gamma, \delta]=(\gamma \delta)^{2}(\gamma \delta)^{-1} \gamma^{-2}(\gamma \delta) \delta^{-2}
$$

Applying the antihomomorphism $\Psi$ to the left-hand side, we obtain the commutator $\left[T_{a}, T_{b}\right]$. Applying $\Psi$ to the right-hand side and using the above descriptions of $\Psi(\gamma), \Psi(\delta)$, and $\Psi(\gamma \delta)$ in terms of Dehn twists (and remembering that $\Psi$ is an antihomomorphism), we obtain

$$
\Psi(\delta)^{-2} \Psi(\gamma \delta) \Psi(\gamma)^{-2} \Psi(\gamma \delta)^{-1} \Psi(\gamma \delta)^{2}=T_{a}^{2}\left(T_{c} T_{e}^{-1}\right) T_{b}^{2}\left(T_{c} T_{e}^{-1}\right)^{-1}\left(T_{c} T_{e}^{-1}\right)^{2}
$$

Using now the formula $f T_{b} f^{-1}=T_{f(b)}$, the fact that $T_{c} T_{e}^{-1}(b)=d$, and the fact that $T_{c}$ and $T_{e}$ commute we see that the right-hand side is equal to $T_{a}^{2} T_{d}^{2} T_{c}^{2} T_{e}^{-2}$. The lemma follows.

Proposition 4.3. For $n \geq 3$, the commutator subgroup $\mathrm{PB}_{n}^{\prime}$ of $\mathrm{PB}_{n}$ is normally generated in $\mathrm{B}_{n}$ by the single element $\left[a_{12}, a_{13}\right]$.

Proof. First, the commutator subgroup of any group is normally generated in that group by the commutators of the generators. Thus $\mathrm{PB}_{n}^{\prime}$ is normally generated in $\mathrm{PB}_{n}$ by all of the commutators $\left[a_{i j}, a_{k \ell}\right]$.

$\operatorname{Next}, \operatorname{Mod}\left(D_{n}\right)$ acts on the set of ordered pairs of distinct curves $\left(c_{i j}, c_{k \ell}\right)$ with three orbits, corresponding to whether the curves have geometric intersection number equal to 0,2 , or 4 . These orbits are represented by the pairs 
$\left(c_{12}, c_{34}\right),\left(c_{12}, c_{13}\right)$, and $\left(c_{13}, c_{24}\right)$, respectively. It follows that the action of $\mathrm{B}_{n}$ on the set of ordered pairs of Artin generators $a_{i j}$ has three orbits, represented by $\left(a_{12}, a_{34}\right),\left(a_{12}, a_{13}\right)$, and $\left(a_{12}, a_{34}\right)$. As the commutator $\left[a_{12}, a_{34}\right]$ is trivial, it follows that $\mathrm{PB}_{n}^{\prime}$ is normally generated in $\mathrm{B}_{n}$ by $\left[a_{12}, a_{23}\right]$ and $\left[a_{13}, a_{24}\right]$.

We have the following relation in $\mathrm{PB}_{n}$ :

$$
\left[a_{13}, a_{24}\right]=\left(a_{13} a_{23}^{-1}\right)\left[a_{23}, a_{24}\right]\left(a_{13} a_{23}^{-1}\right)^{-1} a_{23}^{-1}\left[a_{24}, a_{23}\right] a_{23}
$$

(this relation is obtained by expanding the well-known relator $\left[a_{23} a_{13} a_{23}^{-1}, a_{24}\right]$ for $\mathrm{PB}_{n}$ via the Witt-Hall relation $\left.[x y, z]=x[y, z] x^{-1}[x, z]\right)$. By the previous paragraph, this relation equates $\left[a_{13}, a_{24}\right]$ with a product of two conjugates in $\mathrm{B}_{n}$ of $\left[a_{12}, a_{13}\right]$. This completes the proof.

Forgetful maps. For any $n$ and any $0 \leq k \leq n$ there are $\left(\begin{array}{l}n \\ k\end{array}\right)$ forgetful maps $\mathrm{PB}_{n} \rightarrow \mathrm{PB}_{k}$ obtained by forgetting $n-k$ strands. The various forgetful maps $\mathrm{PB}_{n} \rightarrow \mathrm{PB}_{2} \cong \mathbb{Z}$ are the coordinates of a surjective homomorphism $\mathrm{PB}_{n} \rightarrow$ $\left.\mathbb{Z}^{(} \begin{array}{l}n \\ 2\end{array}\right)$ which is in fact the abelianization of $\mathrm{PB}_{n}$. At the other extreme, the kernel of any forgetful map $\mathrm{PB}_{n} \rightarrow \mathrm{PB}_{n-1}$ corresponds to the image of a push map, so there is a short exact sequence:

$$
1 \rightarrow \pi_{1}\left(D_{n}^{\prime}, p\right) \rightarrow \mathrm{PB}_{n} \rightarrow \mathrm{PB}_{n-1}
$$

(this is a special case of the so-called Birman exact sequence). This exact sequence has an obvious splitting.

Proof of Proposition 4.1. Since $\mathrm{PB}_{n}^{2}$ equals the kernel of the mod two abelianization of $\mathrm{PB}_{n}$, it follows that $\mathrm{PB}_{n}^{2}$ is the preimage of $2 \mathbb{Z}\left(\begin{array}{l}n \\ 2\end{array}\right)$ under the abelianization map

$$
\alpha: \mathrm{PB}_{n} \rightarrow \mathbb{Z}^{\left(\begin{array}{l}
n \\
2
\end{array}\right)} .
$$

As above, the $\left(\begin{array}{l}n \\ 2\end{array}\right)$ coordinates of $\alpha$ are given by the various forgetful maps $\mathrm{PB}_{n} \rightarrow \mathrm{PB}_{2} \cong \mathbb{Z}$. The group $\mathrm{PB}_{2}$ is generated by a Dehn twist. It follows that $\alpha\left(\mathcal{T}_{n}[2]\right)$ is precisely $2 \mathbb{Z}\left(\begin{array}{l}n \\ 2\end{array}\right)$, and so $\mathcal{T}_{n}[2]$ has the same image under $\alpha$ as $\mathrm{PB}_{n}^{2}$. It remains to show that $\mathcal{T}_{n}[2]$, like $\mathrm{PB}_{n}^{2}$, contains the kernel of $\alpha$, namely, the commutator subgroup $\mathrm{PB}_{n}^{\prime}$ of $\mathrm{PB}_{n}$.

By Proposition $4.2, \mathcal{T}_{n}[2]$ contains the commutator $\left[a_{12}, a_{13}\right]$ (see the discussion after the statement). As $\mathcal{T}_{n}[2]$ is normal in $\mathrm{B}_{n}$ it then follows from Proposition 4.3 that $\mathcal{T}_{n}[2]$ contains $\mathrm{PB}_{n}^{\prime}$, as desired.

We pause to record the following corollary of the Main Theorem. In the statement, $\mathcal{B I}_{n}$ is the kernel of the integral Burau representation of $\mathrm{B}_{n}$.

Corollary 4.4. Let $\mathcal{B I}_{n} \leqslant H \leqslant \mathrm{~B}_{n}[4]$. For any $1 \leq k<n$, the image of $H$ under any forgetful map $\mathrm{PB}_{n} \rightarrow \mathrm{PB}_{k}$ is $\mathrm{B}_{k}[4]$.

Proof. Let $F: \mathrm{PB}_{n} \rightarrow \mathrm{PB}_{k}$ be a forgetful map. Clearly $F$ preserves squares of Dehn twists, so we have $F\left(\mathcal{T}_{n}[2]\right) \subseteq \mathcal{T}_{k}[2]$. Hence by the Main Theorem we have that $F\left(\mathrm{~B}_{n}[4]\right) \subseteq \mathrm{B}_{k}[4]$, and in particular $F(H) \subseteq \mathrm{B}_{k}[4]$. 
For the other containment, let $T_{c}^{2} \in \mathrm{B}_{k}$. By the Main Theorem, squares of Dehn twists generate $\mathrm{B}_{k}[4]$ and so it suffices to show that $T_{c}^{2}$ lies in the image of $F$. We can choose a curve $\tilde{c} \subseteq D_{n}$ so that $\tilde{c}$ contains an odd number of marked points and so that $\tilde{c}$ maps to $c$ under the forgetful map $D_{n} \rightarrow D_{k}$ (if $c$ does not already surround an odd number of points, we "remember" one marked point inside $c$ ). Then $F\left(T_{\tilde{c}}^{2}\right)=T_{c}^{2}$. Moreover, $T_{\tilde{c}}^{2}$ lies in $\mathcal{B} \mathcal{I}_{n}$ as its lift to $\operatorname{SMod}\left(S_{g}^{1}\right)$ or $\operatorname{SMod}\left(S_{g}^{2}\right)$ is a Dehn twist about a separating curve. Thus, $T_{\tilde{c}}^{2}$ lies in $H$ by assumption. The corollary follows.

\section{Burau images of Point pushing subgroups}

Denote the $n$ marked points of $D_{n}$ by $p_{1}, \ldots, p_{n}$. As in Section 4 , for each $1 \leq i \leq n$ there is a point pushing subgroup $\pi_{1}\left(D_{n}^{\prime}, p_{i}\right) \subseteq \operatorname{Mod}\left(D_{n}\right)$. For any $1 \leq k \leq n$ we define $\mathrm{K}_{n, k}$ to be the subgroup of $\mathrm{PB}_{n}$ corresponding to the intersection

$$
\pi_{1}\left(D_{n}^{\prime}, p_{1}\right) \cap \cdots \cap \pi_{1}\left(D_{n}^{\prime}, p_{k}\right)
$$

The group $\mathrm{K}_{n, n}$ is the Brunnian subgroup $\mathrm{Brun}_{n}$ of $\mathrm{PB}_{n}$, that is, the subgroup consisting of the braids that become trivial when any one strand is deleted. In this section we prove the following proposition; the $k=1$ case of the first statement appears in the paper of $\mathrm{Yu}$ [34, Theorem 7.3(iii)].

Theorem 5.1. Let $g \geq 2$.

(1) For $1 \leq k \leq 2 g+1, \quad \rho\left(\mathrm{K}_{2 g+1, k}\right)$ contains $\operatorname{Sp}_{2 g}(\mathbb{Z})[4]$ and

$$
\rho\left(\mathrm{K}_{2 g+1, k}\right) / \mathrm{Sp}_{2 g}(\mathbb{Z})[4] \cong \begin{cases}(\mathbb{Z} / 2)^{2 g} & k=1 \\ \mathbb{Z} / 2 & k=2 \\ 1 & k \geq 3 .\end{cases}
$$

(2) For $1 \leq k \leq 2 g+2, \quad \rho\left(\mathrm{K}_{2 g+2, k}\right)$ contains $\left(\operatorname{Sp}_{2 g}(\mathbb{Z})[4]\right)_{\vec{y}_{g+1}}$ and

$$
\rho\left(\mathrm{K}_{2 g+2, k}\right) /\left(\operatorname{Sp}_{2 g}(\mathbb{Z})[4]\right)_{\vec{y}_{g+1}} \cong \begin{cases}(\mathbb{Z} / 2)^{2 g+1} & k=1 \\ \mathbb{Z} / 2 & k=2 \\ 1 & k \geq 3 .\end{cases}
$$

One can use Theorem 5.1 to compute the indices of $\rho\left(\mathrm{K}_{2 g+1, k}\right)$ and $\rho\left(\mathrm{K}_{2 g+2, k}\right)$ in $\operatorname{Sp}_{2 g}(\mathbb{Z})[2]$ and $\left(\mathrm{Sp}_{2 g}(\mathbb{Z})[2]\right)_{\vec{y}_{g+1}}$, respectively, using the facts that

$$
\left[\mathrm{Sp}_{2 g}(\mathbb{Z})[2]: \operatorname{Sp}_{2 g}(\mathbb{Z})[4]\right]=2^{\left(\begin{array}{c}
2 g+1 \\
2
\end{array}\right)}
$$

and

$$
\left[\left(\mathrm{Sp}_{2 g}(\mathbb{Z})[2]\right)_{\vec{y}_{g+1}}:\left(\mathrm{Sp}_{2 g}(\mathbb{Z})[4]\right)_{\vec{y}_{g+1}}\right]=2^{\left(\begin{array}{c}
2 g+2 \\
2
\end{array}\right)} .
$$

For example:

$$
\left[\mathrm{Sp}_{2 g}(\mathbb{Z})[2]: \rho\left(\mathrm{K}_{2 g+1,1}\right)\right]=2^{g(2 g+1)}-2^{2 g} .
$$

It also follows immediately from Theorem 5.1 that

(1) $\rho\left(\operatorname{Brun}_{2 g+1}\right)=\operatorname{Sp}_{2 g}(\mathbb{Z})[4]$ and

(2) $\rho\left(\operatorname{Brun}_{2 g+2}\right)=\left(\mathrm{Sp}_{2 g}(\mathbb{Z})[4]\right)_{\vec{y}_{g+1}}$. 
We will require the following theorem. The first statement is due to Mennicke [23, Section 10] and the second statement follows easily from the first statement and the same type of considerations as in the proof of Theorem 3.3.

Theorem 5.2. Let $g \geq 2$ and let $m \geq 2$.

(1) $\mathrm{Sp}_{2 g}(\mathbb{Z})[m]$ is generated by $\left\{\tau_{\vec{v}}^{m} \mid \vec{v} \in \mathbb{Z}^{2 g}\right.$ primitive $\}$.

(2) $\left(\operatorname{Sp}_{2 g+2}(\mathbb{Z})[4]\right)_{\vec{y}_{g+1}}$ is generated by $\left\{\tau_{\vec{v}}^{m} \mid \vec{v} \in \mathbb{Z}^{2 g+2}\right.$ primitive,

$$
\left.\hat{\imath}\left(\vec{v}, \vec{y}_{g+1}\right)=0\right\} \text {. }
$$

Say that a simple closed curve $c$ in $S_{g}^{1}$ is pre-symmetric if $\iota(c) \cap c=\emptyset$.

Proposition 5.3. If $\vec{v} \in H_{1}\left(X_{n} ; \mathbb{Z}\right)$ is primitive then it is represented by a pre-symmetric, oriented simple closed curve.

Proof. We first treat the case $n=2 g+1$, in which case $X_{n} \cong S_{g}^{1}$. It follows from the description of Arnol'd's isomorphism between $H_{1}\left(S_{g}^{1} ; \mathbb{Z} / 2\right)$ and $H_{1}\left(D_{2 g+1}^{\circ} ; \mathbb{Z} / 2\right)^{\text {even }}$ that there is a pre-symmetric representative $c^{\prime}$ in $S_{g}^{1}$ of the mod two reduction of $\vec{v}$. Indeed, any class in $H_{1}\left(D_{2 g+1}^{\circ} ; \mathbb{Z} / 2\right)^{\text {even }}$ is represented by a simple closed curve surrounding an even number of marked points, and the preimage of such a curve has two components, either of which is the desired $c^{\prime}$.

Let $\vec{v}^{\prime}$ denote the class of $c^{\prime}$ in $H_{1}\left(S_{g}^{1} ; \mathbb{Z}\right)$. The group $\operatorname{Sp}_{2 g}(\mathbb{Z})[2]$ acts transitively on the representatives of a given class in $H_{1}\left(S_{g}^{1} ; \mathbb{Z} / 2\right)$ (see, e.g. [7, Corollary 3.11]) and so there is an $M \in \operatorname{Sp}_{2 g}(\mathbb{Z})[2]$ with $M\left(\vec{v}^{\prime}\right)=\vec{v}$. By Theorem 3.3(1), there is a $b \in \mathrm{PB}_{2 g+1}$ with $\rho(b)=M$. If $\tilde{b}$ is the corresponding element of $\operatorname{SMod}\left(S_{g}^{1}\right)$, then $\tilde{b}\left(c^{\prime}\right)$ is the desired representative.

The case of $n=2 g+2$ is almost exactly the same. The main difference is that we must choose $M$ to lie in $\left(\mathrm{Sp}_{2 g+2}(\mathbb{Z})[2]\right)_{\vec{y}_{g+1}}$ (the existence of such an $M$ follows by applying the same statement as before [7, Corollary 3.11] to the pair $\left.\vec{v}, \vec{y}_{g+1}\right)$. We can then apply Theorem 3.3(2) to complete the proof.

One can prove Proposition 5.3 without Theorem 3.3 by applying a hyperelliptic version of the Euclidean algorithm for simple closed curves due to Meeks and Patrusky (see [13, Proposition 6.2]).

Symmetric homology classes. We remark that the pre-symmetric representative of $\vec{v}$ given by Proposition 5.3 is homotopic to a symmetric one (that is, one fixed by the hyperelliptic involution) if and only if the corresponding element of $H_{1}\left(D_{2 g+1}^{\circ} ; \mathbb{Z} / 2\right)^{\text {even }}$ has exactly two nonzero entries in the standard basis, that is, if and only if the corresponding simple closed curve in $D_{n}$ surrounds exactly two marked points. In particular, the existence of a symmetric representative of $\vec{v}$ is completely determined by the mod two reduction of $\vec{v}$. This was observed by A'Campo [1, Théorème 3]; see also Wajnryb [32, Theorem 2 and Corollary]. 
Proof of Theorem 5.1. We begin with the first statement, which concerns the odd-stranded braid groups $\mathrm{PB}_{2 g+1}$ with $g \geq 2$. The first goal is to prove that $\rho\left(\mathrm{K}_{2 g+1, k}\right)$ contains $\mathrm{Sp}_{2 g}(\mathbb{Z})[4]$. To do this, it is enough to show $\rho\left(\mathrm{K}_{2 g+1,1}\right)$ contains $\mathrm{Sp}_{2 g}(\mathbb{Z})[4]$, as the subgroups of $\mathrm{PB}_{2 g+1}$ corresponding to the $\pi_{1}\left(D_{2 g+1}^{\prime}, p_{i}\right)$ are conjugate in $\mathrm{B}_{2 g+1}$ and $\mathrm{Sp}_{2 g}(\mathbb{Z})[4]$ is normal in $\mathrm{Sp}_{2 g}(\mathbb{Z})$.

By Theorem 5.2(1), it is enough to show that $\rho\left(\mathrm{K}_{2 g+1,1}\right)$ contains every $\tau_{\vec{v}}^{4}$ with $\vec{v}$ primitive. To this end, let $\vec{v}$ be a primitive element of $\mathbb{Z}^{2 g}$. By Proposition 5.3, there is a pre-symmetric representative $c$ of $\vec{v}$ in $S_{g}^{1}$.

Let $\bar{c}$ denote the image of $c$ in $D_{2 g+1}$; the curve $\bar{c}$ is a simple closed curve surrounding an even number of marked points. Choose a simple closed curve $\bar{d}$ in $D_{2 g+1}$ so that $\bar{c} \cup \bar{d}$ form the boundary of an annulus containing the marked point $p_{1}$ (and no other $p_{i}$ ).

Clearly the element of $\mathrm{PB}_{2 g+1}$ given by $T_{\bar{c}}^{2} T_{\bar{d}}^{-2}$ lies in $\mathrm{K}_{2 g+1,1}$. We claim that it maps to $\tau_{\vec{v}}^{4}$ under $\rho$. The image of $T_{\bar{c}}^{2}$ in $\operatorname{Mod}\left(S_{g}^{1}\right)$ is $T_{c}^{2} T_{\iota(c)}^{2}$ and the image of the latter in $\operatorname{Sp}_{2 g}(\mathbb{Z})$ is $\tau_{[\vec{c}]}^{2} \tau_{[\iota(\vec{c})]}^{2}=\tau_{\vec{v}}^{4}$ (here we choose an arbitrary orientation on $c)$. Next, since $\bar{d}$ surrounds an odd number of marked points, the image of $T_{\bar{d}}^{2}$ in $\operatorname{Mod}\left(S_{g}^{1}\right)$ is a Dehn twist about a separating curve and the image of the latter in $\mathrm{Sp}_{2 g}(\mathbb{Z})$ is trivial. This gives the claim, and hence the statement that $\rho\left(\mathrm{K}_{2 g+1, k}\right)$ contains $\mathrm{Sp}_{2 g}(\mathbb{Z})[4]$.

We now proceed to compute the image of $\mathrm{K}_{2 g+1, k}$ in $\operatorname{Sp}_{2 g}(\mathbb{Z})[2] / \operatorname{Sp}_{2 g}(\mathbb{Z})[4]$. Recall from Section 3 that $\mathrm{Sp}_{2 g}(\mathbb{Z})[2] / \mathrm{Sp}_{2 g}(\mathbb{Z})[4]$ is isomorphic to $\left.(\mathbb{Z} / 2)^{\left({ }^{2 g+1}\right.}{ }^{2}\right)$ and the coordinates of the resulting map $\left.\mathrm{PB}_{2 g+1} \rightarrow(\mathbb{Z} / 2)^{(2 g+1}{ }_{2}\right)$ are given by the various maps $f_{i j}: \mathrm{PB}_{2 g+1} \rightarrow \mathrm{PB}_{2} / \mathrm{PB}_{2}^{2}$ obtained by forgetting all strands except the $i$ th and $j$ th and reducing modulo two.

We claim that $f_{i j}\left(\mathrm{~K}_{2 g+1, k}\right)$ is nontrivial if and only if $\{1, \ldots, k\} \subseteq\{i, j\}$. Indeed, if $\{1, \ldots, k\} \subseteq\{i, j\}$ then the pure braid $a_{i j}$ from Section 4 lies in $\mathrm{K}_{2 g+1, k}$ and has nontrivial image under $f_{i j}$. On the other hand if $\{1, \ldots, k\} \nsubseteq$ $\{i, j\}$ then $f_{i j}\left(\mathrm{~K}_{2 g+1, k}\right)$ is trivial by definition, giving the claim. The description of the image of $\mathrm{K}_{2 g+1, k}$ follows immediately.

The theorem for the even-stranded braid group is proven in the same way, with $\mathrm{PB}_{2 g+1}, \mathrm{~K}_{2 g+1, k}$, and $\mathrm{Sp}_{2 g}(\mathbb{Z})[2]$ replaced with $\mathrm{PB}_{2 g+2}, \mathrm{~K}_{2 g+2, k}$, and $\left(\mathrm{Sp}_{2 g}(\mathbb{Z})[2]\right)_{\vec{y}_{g+1}}$ and with Theorem 5.2(1) replaced by Theorem 5.2(2).

Theorem 5.2 also holds in the case $g=1$ and $m=4$. To see this, consider the action of $\mathrm{Sp}_{2}(\mathbb{Z})[4]=\mathrm{SL}_{2}(\mathbb{Z})[4]$ on the hyperbolic plane $\mathbb{H}^{2}$. Using the Farey tessellation of $\mathbb{H}^{2}$, the quotient is easily seen to be a punctured sphere (it is an octahedron minus the vertices) and so the fundamental group is generated by loops around the punctures. Each of these corresponds to a fourth power of a transvection.

Hence, our proof of Theorem 5.1 also applies in the case of $g=1$ and so $\rho\left(\operatorname{Brun}_{3}\right)=\mathrm{SL}_{2}(\mathbb{Z})[4]$. The group $\mathcal{B I}_{3}$ is contained in the center of $\mathrm{B}_{3}$, which has trivial intersection with $\mathrm{Brun}_{3}$. It follows that:

$$
\operatorname{Brun}_{3} \cong \mathrm{SL}_{2}(\mathbb{Z})[4] \text {. }
$$


A version of this isomorphism was previously observed by Cohen and $\mathrm{Wu}$ $[9]$.

\section{REFERENCES}

[1] Norbert A'Campo. Tresses, monodromie et le groupe symplectique. Comment. Math. Helv., 54(2):318-327, 1979.

[2] V. I. Arnol'd. A remark on the branching of hyperelliptic integrals as functions of the parameters. Funkcional. Anal. i Priložen., 2(3):1-3, 1968.

[3] Mamoru Asada. The faithfulness of the monodromy representations associated with certain families of algebraic curves. J. Pure Appl. Algebra, 159(2-3):123-147, 2001.

[4] Joachim Assion. A proof of a theorem of Coxeter. C. R. Math. Rep. Acad. Sci. Canada, 1(1):41-44, 1978/79.

[5] Gavin Band and Philip Boyland. The Burau estimate for the entropy of a braid. Algebr. Geom. Topol., 7:1345-1378, 2007.

[6] Joan S. Birman and Hugh M. Hilden. On the mapping class groups of closed surfaces as covering spaces. In Advances in the theory of Riemann surfaces (Proc. Conf., Stony Brook, N.Y., 1969), pages 81-115. Ann. of Math. Studies, No. 66. Princeton Univ. Press, Princeton, N.J., 1971.

[7] Tara Brendle, Dan Margalit, and Andrew Putman. Generators for the hyperelliptic Torelli group and the kernel of the Burau representation at $t=-1$. to appear in Inventiones Mathematicae.

[8] Thomas Church and Andrew Putman. Generating the Johnson filtration. preprint.

[9] F. R. Cohen and J. Wu. On braid groups and homotopy groups. In Groups, homotopy and configuration spaces, volume 13 of Geom. Topol. Monogr., pages 169-193. Geom. Topol. Publ., Coventry, 2008.

[10] H. S. M. Coxeter. Factor groups of the braid group. Proc. Fourth Canadian Math. Congress, Banff, pages 95-122, 1957.

[11] P. Deligne and G. D. Mostow. Monodromy of hypergeometric functions and nonlattice integral monodromy. Inst. Hautes Études Sci. Publ. Math., (63):5-89, 1986.

[12] Steven Diaz, Ron Donagi, and David Harbater. Every curve is a Hurwitz space. Duke Math. J., 59(3):737-746, 1989.

[13] Benson Farb and Dan Margalit. A primer on mapping class groups, volume 49 of Princeton Mathematical Series. Princeton University Press, Princeton, NJ, 2012.

[14] Louis Funar and Toshitake Kohno. On Burau's representations at roots of unity. Geom. Dedicata, 169:145-163, 2014.

[15] Jean-Marc Gambaudo and Étienne Ghys. Braids and signatures. Bull. Soc. Math. France, 133(4):541-579, 2005.

[16] Richard Hain. Finiteness and Torelli spaces. In Problems on mapping class groups and related topics, volume 74 of Proc. Sympos. Pure Math., pages 57-70. Amer. Math. Soc., Providence, RI, 2006.

[17] Stephen P. Humphries. Normal closures of powers of Dehn twists in mapping class groups. Glasgow Math. J., 34(3):313-317, 1992.

[18] Stephen P. Humphries. Subgroups of pure braid groups generated by powers of Dehn twists. Rocky Mountain J. Math., 37(3):801-828, 2007.

[19] Mikhail Khovanov and Paul Seidel. Quivers, Floer cohomology, and braid group actions. J. Amer. Math. Soc., 15(1):203-271, 2002.

[20] Wilhelm Magnus and Ada Peluso. On a theorem of V. I. Arnol'd. Comm. Pure Appl. Math., 22:683-692, 1969.

[21] Curtis T. McMullen. Braid groups and Hodge theory. Math. Ann., 355(3):893-946, 2013.

[22] D. B. McReynolds. The congruence subgroup problem for pure braid groups: Thurston's proof. New York J. Math., 18:925-942, 2012. 
[23] J. Mennicke. Zur Theorie der Siegelschen Modulgruppe. Math. Ann., 159:115-129, 1965.

[24] Takayuki Morifuji. On Meyer's function of hyperelliptic mapping class groups. J. Math. Soc. Japan, 55(1):117-129, 2003.

[25] David Mumford. Tata lectures on theta. I, volume 28 of Progress in Mathematics. Birkhäuser Boston, Inc., Boston, MA, 1983. With the assistance of C. Musili, M. Nori, E. Previato and M. Stillman.

[26] David Mumford. Tata lectures on theta. II, volume 43 of Progress in Mathematics. Birkhäuser Boston, Inc., Boston, MA, 1984. Jacobian theta functions and differential equations, With the collaboration of C. Musili, M. Nori, E. Previato, M. Stillman and $\mathrm{H}$. Umemura.

[27] M. Newman and J. R. Smart. Symplectic modulary groups. Acta Arith, 9:83-89, 1964.

[28] Andrew Putman. The Picard group of the moduli space of curves with level structures. Duke Math. J., 161(4):623-674, 2012.

[29] N. F. Smythe. The Burau representation of the braid group is pairwise free. Arch. Math. (Basel), 32(4):309-317, 1979.

[30] William P. Thurston. Shapes of polyhedra and triangulations of the sphere. In The Epstein birthday schrift, volume 1 of Geom. Topol. Monogr., pages 511-549. Geom. Topol. Publ., Coventry, 1998.

[31] T. N. Venkataramana. Image of the Burau representation at $d$-th roots of unity. Ann. of Math. (2), 179(3):1041-1083, 2014.

[32] Bronislaw Wajnryb. On the monodromy group of plane curve singularities. Math. Ann., 246(2):141-154, 1979/80.

[33] Bronislaw Wajnryb. A braidlike presentation of $\operatorname{Sp}(n, p)$. Israel J. Math., 76(3):265288, 1991.

[34] Jiu-Kang Yu. Toward a proof of the Cohen-Lenstra conjecture in the function field case. Preprint.

Tara E. Brendle, School of Mathematics \& Statistics, 15 University GarDENS, University of Glasgow, G12 8QW, TARA.Brendle@GLAsGow.AC.UK

Dan Margalit, School of Mathematics, Georgia Institute of Technology, 686 Cherry St., Atlanta, GA 30332, margalit@math.Gatech.edu 